\title{
Piezo-driven valve for disruption mitigation studies in tokamaks
}

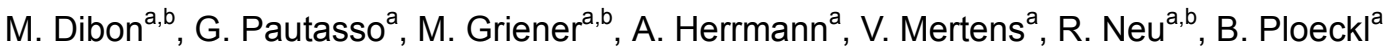 \\ ${ }^{a}$ Max-Planck-Institute for Plasma Physics, Boltzmannstr. 2, 85748 Garching, Germany \\ ${ }^{b}$ Technical University Munich, Boltzmannstr. 15, 85748 Garching, Germany
}

Disruptions are a major issue for the safe operation of tokamaks. Hence the mitigation of disruptive effects is of high priority in view of ITER and DEMO. Previous experiments at ASDEX Upgrade have shown that the injections of massive amounts of gaseous impurities using in-vessel valves mitigate disruptions very efficiently, due to short time of flight of the gas towards the plasma edge and the applicability of pure noble gases. A new valve concept for in-vessel massive gas injection has been developed at ASDEX Upgrade to replace the old valves which suffered from reliability problems. This new valve is a normally closed valve since the valve plate is pressed into the main sealing by a stainless steel bellow (acting as a spring), sealing off the gas reservoir, which can hold $42 \mathrm{~cm}^{3}$ of gas at a pressure of up to $5 \mathrm{MPa}$. The valves actuator consists of 4 piezoelectric stacks which are mounted in two parallel pairs into a monolithic titanium frame. This frame is rigidly connected to the valve plate and it amplifies the stroke of the stacks to allow a total stroke of the valve plate of $2.2 \mathrm{~mm}$, which is reached after $5 \mathrm{~ms}$. The diameter of the straight valve nozzle is $14 \mathrm{~mm}$ allowing a peak flow rate of $44 \mathrm{kPam} / \mathrm{s}$ after $1 \mathrm{~ms}$. The valve has a size of $173 \mathrm{~mm} \times 88 \mathrm{~mm} \times 70 \mathrm{~mm}$.

Keywords: massive gas injection, disruption mitigation

\section{INTRODUCTION}

Disruptions are a serious issue for the safe operation of tokamak fusion devices. Technical errors can cause MHD modes which lead to a sudden loss of the plasma thermal energy. This leads to a disruption in which the divertor is exposed to a significantly higher heat flux than in normal operation. This can cause damages if the plasma thermal energy is high. Additionally, elongated plasmas can move towards the upper or lower divertor during disruptions if their vertical position is perturbed beyond the controllable region. This movement induces eddy currents in conducting structures and drives halo currents ${ }^{1}$. Both currents lead to tremendous forces on the vessel. Further, strong toroidal electric fields can give rise to runaway electrons (RE) with relativistic energies, depending on the plasma density and current. These can cause massive damage to plasma facing components if they lose confinement.

Massive gas injection (MGI) has proven to be an effective method to mitigate the heat loads, forces and REs. This has been observed at several different machines like ASDEX Upgrade ${ }^{2}$ (AUG), Alcator CMod $^{3}$, DIII-D ${ }^{4-6}$, JET $^{7}$, MAST $^{8}$, TEXTOR ${ }^{9}$ and Tore Supra $^{10}$. MGI is performed using fast valves. It was proven favorable to use valves close to the plasma because the assimilation fraction is high ${ }^{11}$. In the last years, spring-driven valves were used for this purpose in AUG, but they suffered from reliability issues. An examination of these old valves revealed problems with the valve seal, the clamping mechanism and the sensitive adjustment of the piezoelectric stacks.

In addition to an improved spring-driven valve ${ }^{12}$ a new piezo-driven valve was developed at AUG as a normally closed counterpart to the spring-driven valves. This paper presents the basic valve design, the design of the active components, especially the piezoelectric actuator and first test results.

\section{VALVE DESIGN}

The valve has to fulfill both physical and technical requirements to be suited for operation. The physical requirements are given by the phenomenology of disruptions as explained before. The pre-TQ is of the order of milliseconds; hence the opening time of the valve must be at least of the same order. The total amount of required impurity atoms has been found to be about $10^{21}$ particles in AUG. The gas reservoir must be designed accordingly. As for the technical requirements, the valve must be suitable for in-vessel conditions. This is first of all compatibility with ultrahigh vacuum, meaning the valve, the seals and gas lines must have a leak rate blow $10^{-8} \mathrm{Pam}^{3} / \mathrm{s}$ and the used materials must not emit gas themselves. Secondly the valve must be insensitive to magnetic fields and must not create any. The valve must withstand the baking temperature of $150{ }^{\circ} \mathrm{C}$ and ionizing radiation without taking damage. The piezodriven valve fulfills these requirements due to its actuation concept and used materials, which are ceramic, titanium, fluoroelastomer (FKM), copper and stainless steel. The composition of the valve with its main components is illustrated in Fig. 1.

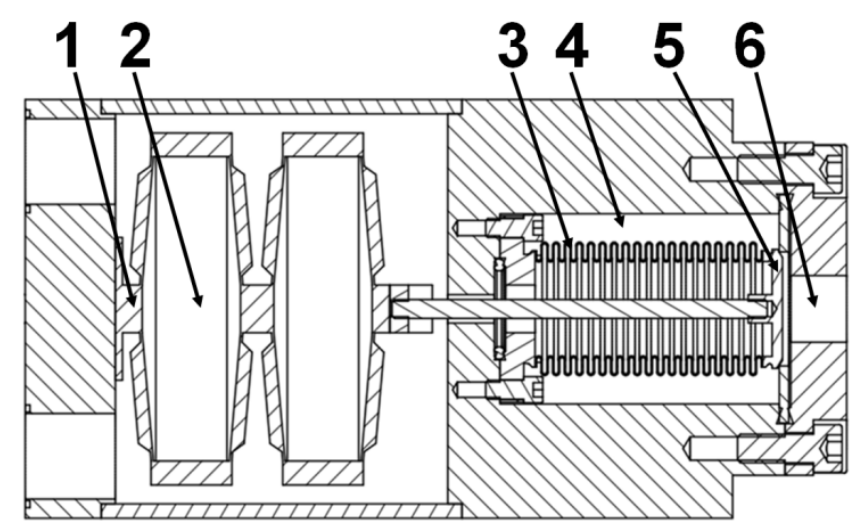

Figure 1: Scheme of the valve with the titanium frame (1), the piezoelectric stack actuators (2), the bellow (3), the gas reservoir (4), the valve plate (5) and the nozzle (6) 
The piezo-driven valve is a normally-closed valve, meaning, that in the idle state the conical valve plate (5) is pressed into the FKM seal by the bellow (3) closing off the gas reservoir (4). The reservoir can hold $42 \mathrm{~cm}^{3}$ of gas at a maximum pressure of $5 \mathrm{MPa}$. This corresponds to a maximum particle inventory of $5 \times 10^{22}$ atoms assuming the gas is at room temperature. When the reservoir is filled and the valve is triggered, the piezoelectric stack actuators (2) are supplied with a voltage of $200 \mathrm{~V}$. The stacks expand $0.1 \mathrm{~mm}$ and the titanium frame (1) amplifies the stroke by a factor of 14. The valve plate is directly connected to the frame, allowing a fast movement of $1.4 \mathrm{~mm}$. The inertia of the stacks and the frame add to the nominal stroke which reaches its maximum at $2.1 \mathrm{~mm}$. The gas flows from the reservoir through the straight nozzle (6), which has a circular orifice of $14 \mathrm{~mm}$ diameter.

\section{A. Piezoelectric actuator}

The piezoelectric actuator consists of 4 piezoelectric stack actuators and a monolithic titanium frame. The stacks are of the type Noliac NAC2023-H64 ${ }^{13}$, meaning they have a length of $64 \mathrm{~mm}$, corresponding to a stroke of $0.1 \mathrm{~mm}$, and a quadratic cross-section with $225 \mathrm{~mm}^{2}$, corresponding to a blocking force of $9450 \mathrm{~N}$ each, when supplied with $200 \mathrm{~V}$ DC. They are mounted into the frame, which consists of two connected rhomb shaped sections, each holding two parallel stacks. The frame is manufactured from a solid block of Ti6Al4V. The material was chosen because of its unique properties with respect to elasticity and tensile strength and the monolithic design guaranties UHV compatibility, as well as heat and wear resistance. The frame serves two purposes: it prestresses the piezoelectric stacks with $150 \mathrm{~N}$ each and it amplifies the stroke of the stacks significantly. The pre-stress is necessary to keep the stacks under compression stress for all conditions. Without this counteracting force, the stacks could delaminate due to their own inertial force when they are charged and expand. The amplification is achieved by geometric deformation of the frame. It can be derived from trigonometric relations. Fig. 2 illustrates how the amplification is achieved using one leg of the titanium frame. The solid rectangular triangle indicates the leg in the original state while the dashed triangle shows the deformed state.

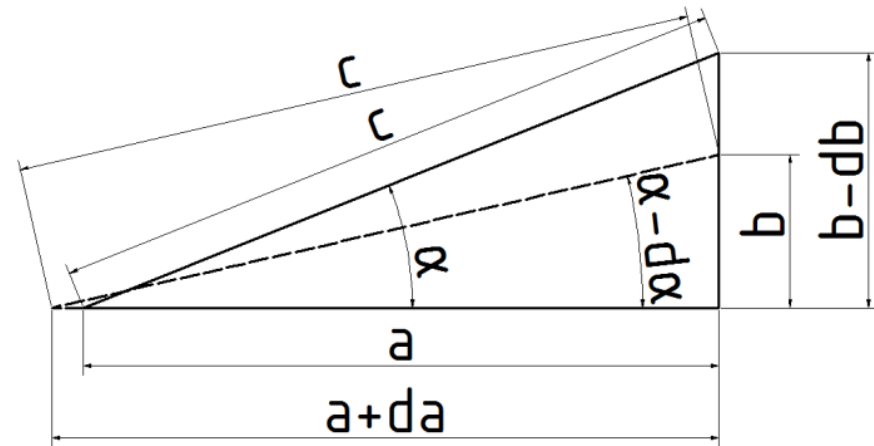

Figure 2: Scheme of one leg of the titanium frame. The solid line indicates the original state and the dashed line the state when the actuators have expanded. a: halflength of a stack, da: half stroke of one stack, $b$ : height of the rhomb, $d b$ : amplified displacement, $a$ : original angle between the leg and the stack, $d \alpha$ : angle variation, $c$ : length of the leg
Relations for the angle variation $d \alpha$ and the displacement $d b$ depending on the half length of the stack $a$ and the half stroke $d a$, which are determined by the piezoelectric stack, and on the original angle $\alpha$ are derived from the trigonometric relations of the triangles shown in fig. 2 .

$$
\begin{aligned}
& \tan d \alpha(a, d a, \alpha)=\frac{\sqrt{a^{2} \tan ^{2} \alpha-2 a d a-d a^{2}}-(a+d a) \tan \alpha}{\sqrt{a^{2} \tan ^{2} \alpha-2 a d a-d a^{2}} \tan \alpha-a-d a} \\
& d b(a, d a, \alpha)=a \tan \alpha-\frac{(\tan \alpha-\tan d \alpha(a, d a, \alpha))(a+d a)}{1-\tan \alpha \tan d \alpha(a, d a, \alpha)}
\end{aligned}
$$

Since the frame consists of two serial rhombs, it has four serial legs that contribute to the stroke amplification. Hence, the overall stroke of the piezoelectric actuator is $4 x d b$.

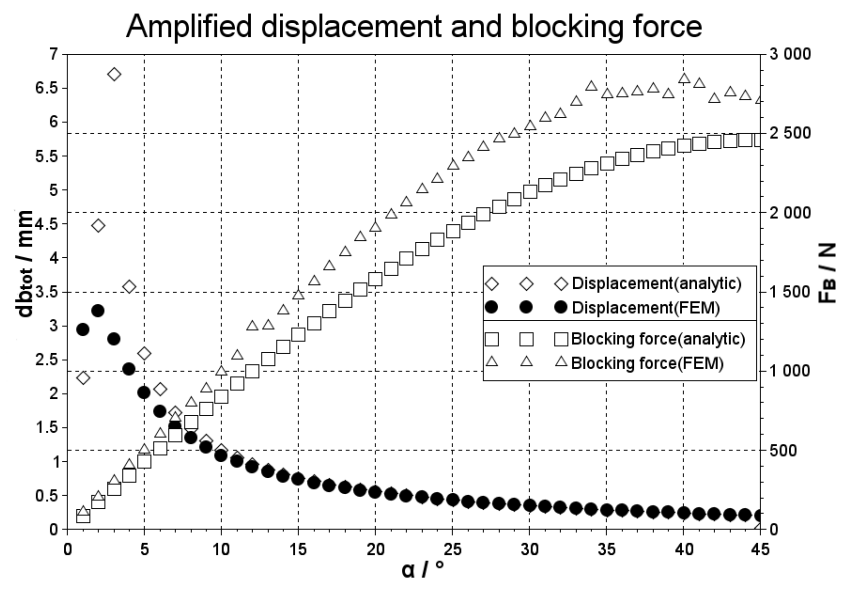

Figure 3: Analytical calculation and finite element model (FEM) simulation of the displacement and the blocking force of an actuator with two rhombs and two stacks in each rhomb. The stacks have a half-length $a=32 \mathrm{~mm}$ and a half stroke $d a=0.05 \mathrm{~mm}$.

The blocking force $F_{B}$ is the force that the actuator produces if it is not allowed to perform any movement. Hence, in this state, only the legs are stretched by the force of the piezoelectric stacks. The blocking force therefore depends on the original angle $\alpha$, and the geometry (mean cross-sectional area $A$, length $c$ ) and material (elasticity $E$ ) of the leg, as well as the blocking force of a single stack $F_{0}$ and the half stroke da of the stacks (Eq. 3).

$$
F_{B}=\frac{1}{2} \sin (2 \alpha) \frac{F_{0}}{d a}\left(d a-\frac{F_{0}}{\frac{F_{0}}{d a}+\frac{E * A}{c}}\right)
$$

The blocking force is independent from the number of serial rhombs in the frame. The linear relation between force and elongation of the stacks is translated by the frame. Thus the actuator has a linear characteristic itself.

The analytic model overestimates the displacement for small angles $\alpha$, while underestimating the blocking force at large angles compared to the FEM simulation. The displacement error is a result of the analytic model neglecting overshoot, meaning the angle variation $d \alpha$ becomes larger than the original angle $\alpha$, leading to an imaginary result for $d b$. The force is slightly underestimated due to averaging over the legs crosssectional area, thus neglecting stress imbalance between the joints and the bulky center of the leg. 


\section{B. Bellow}

The bellow fulfills two functions. Its stiffness presses the valve plate into the main seal, closing the gas reservoir and it relieves the valve plate from the pressure inside the reservoir. The bellow is a Witzenmann BAT $19.8 \times 28 \times 2 \times 0.15^{14}$ with 19 corrugations. The stiffness of this bellow is 22.6 $\mathrm{N} / \mathrm{mm} \pm 6.8 \mathrm{~N} / \mathrm{mm}$. The length of the bellow in the relaxed state is $53 \mathrm{~mm}$ while in the installed state it has a length of $47.9 \mathrm{~mm}$. Taking the uncertainty into account, this results in a minimal pre-stressing force onto the valve seal of $80 \mathrm{~N}$. The total mass of the bellow is $31.3 \mathrm{~g}$ leading to an effective accelerated mass of $10.4 \mathrm{~g}$.

\section{Main valve seal}

The gas reservoir is sealed off by the main valve seal and the valve plate. The seal is a ring shaped FKM plate with an outer diameter of $48 \mathrm{~mm}$ and an inner diameter of $24 \mathrm{~mm}$, with a thickness of $2 \mathrm{~mm}$. This allows the installation into a standard CF40 flange connection. The inner diameter is chosen to fit the hydraulic diameter of the bellow, thus relieving the valve plate from the gas pressure.

\section{Nozzle}

The nozzle is designed to allow the maximum flow rate for the given inlet diameter of $24 \mathrm{~mm}$ while preventing a direct line of sight between the plasma and the FKM seal. A standard CF40 flange with a central drilling of $14 \mathrm{~mm}$ diameter fulfills these requirements. The mass flow rate through the nozzle is governed by the reservoir gas pressure $p_{0}(t)$, the critical cross-sectional area $A(t)$, the reservoir volume $V$, the gas temperature $T_{0}$ and the gas species (adiabatic constant $\gamma$, specific gas constant $R_{s}$ ).

$$
\begin{gathered}
\dot{m}(t)=\frac{p_{0}(t) * \sqrt{\gamma} * A(t)}{\sqrt{R * T_{0}}} *\left(\frac{\gamma+1}{2}\right)^{-\left(\frac{\gamma+1}{2 *(\gamma-1)}\right)} \\
\frac{\partial p_{0}(t)}{\partial t}=\dot{m}(t) * \frac{R * T_{0}}{V}
\end{gathered}
$$

Adiabatic expansion is assumed as the thermal diffusion is slower than the flow. Fast particles are neglected since they play a minor role on the short distance between valve and plasma. As the valve opens, the critical cross-sectional area first is located between the valve plate and the main valve seal, as it is the smallest area. When this cylindrical surface becomes larger, the critical area is shifted to a cylindrical surface between valve plate and nozzle entrance. Time traces of the mass flow rate and the reservoir pressure during the valve opening are shown in Fig. 4, both calculated with the analytical model (Eq. 4 and 5) and a CFD simulation done with ANSYS Fluent. The time before opening of the valve is neglected. The first peak in the CFD mass flow rate is due to the hybrid initialization of the model. This method simulates gas in the nozzle at the onset of the flow, stabilizing the computation but causing this unreal mass flow. The actual peak mass flow rate is about $0.65 \mathrm{~kg} / \mathrm{s}$ for both the analytical model and the CFD simulation. However, the time at which the mass flow reaches its maximum is about $0.5 \mathrm{~ms}$ shorter in the CFD simulation. This is a result of the analytical model neglecting velocity and temperature profiles in the flow. The time traces of the pressures are well aligned, indicating an evacuation time (leaving $5 \%$ of initial reservoir pressure) of 5 to $7 \mathrm{~ms}$.

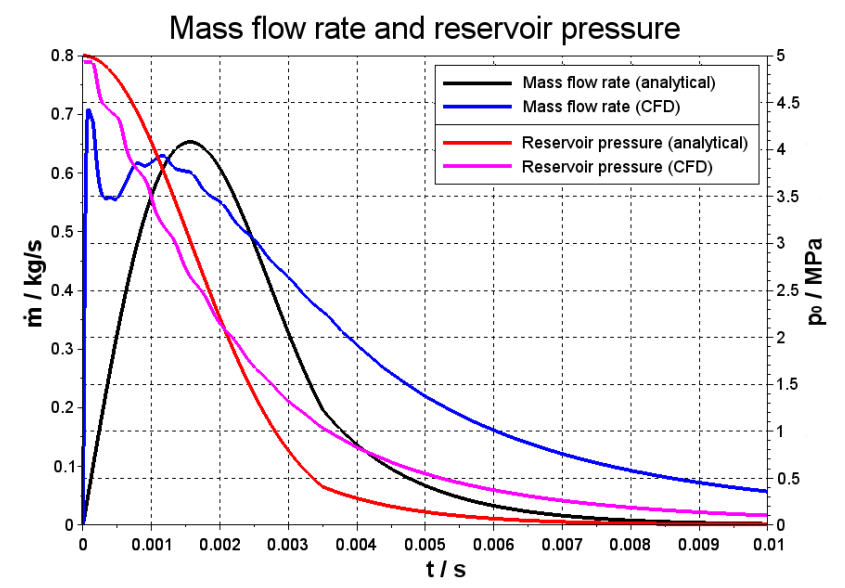

Figure 4: Analytical model and CFD simulation of the mass flow rate and the reservoir pressure for the given nozzle, opening time $=3.5 \mathrm{~ms}, \mathrm{~V}=42 \mathrm{~cm}^{3}, \mathrm{~T}_{0}=300 \mathrm{~K}, \mathrm{p}_{0}$ $=5 \mathrm{MPa}$ and argon as filling gas

\section{TEST RESULTS}

The valve was tested thoroughly before the installation into the vacuum vessel of AUG. This was done to evaluate both the performance and reliability of the valve, which are crucial parameters for a safe operation since access to the valve is limited once AUG restarts operation.

\section{A. Opening time and stroke}

The opening time and the maximal stroke were measured by observing the movement of the valve plate using a fast laser displacement sensor. Laser and valve were therefore fixed onto the same base plate, the laser pointing through the nozzle onto the valve plate. The trigger pulse for the valve was forwarded to a fast oscilloscope which then recorded the analogue signal from the laser. Fig. 5 shows the converted analogue signal using the preset conversion factor of the sensor of $1 \mathrm{~V} / \mathrm{mm}$.

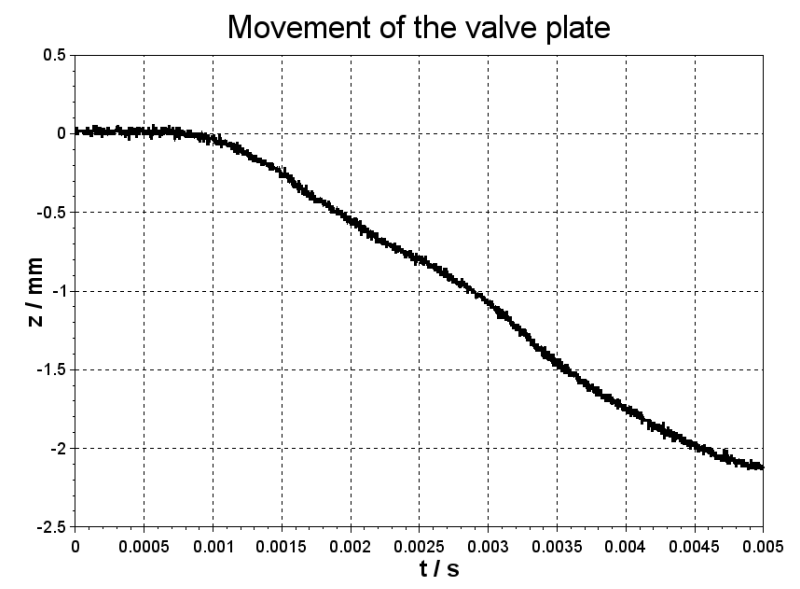

Figure 5: Measured movement of the valve plate over 5 ms after the trigger pulse

The movement starts with a delay of $1 \mathrm{~ms}$ because the piezoelectric stack actuators have to reach a certain charge in order to produce enough force to overcome the pre-stressing force of the bellow and the frame. 
Once the actuator has achieved this necessary force, it pulls the valve plate back with a velocity which is roughly constant at $0.44 \mathrm{~m} / \mathrm{s}$. The plate reaches its maximal stroke of $2.2 \mathrm{~mm}$ after $5 \mathrm{~ms}$. Taking the delay and the minimal stroke of $2 \mathrm{~mm}$ into account which is necessary to achieve the maximal mass flow rate, the overall opening time is $3.5 \mathrm{~ms}$, which is fast enough for disruption mitigation experiments as the bulk of the gas is ejected within this time (fig. 4) and the prethermal quench time is of the same order ${ }^{16}$ for the amount of injected gas by this valve.

\section{B. Gas expansion}

The expansion cloud of the gas coming from the valve nozzle determines the region of the plasma where the strongest cooling takes place during the first milliseconds of MGI. This is important information for numerical models dealing with interaction of plasma and neutrals in the edge (SOLPS, JOREK). For this purpose, the opening angles of the gas cloud have to be determined. A helium glow plasma was used to visualize the gas. Therefore the valve was placed in a vacuum tank with a pumped volume of $0.5 \mathrm{~m}^{3}$ which was then filled with helium at a pressure of $1.3 \mathrm{~Pa}$. The plasma was driven using a voltage of $240 \mathrm{~V}$ creating a plasma current of $1 \mathrm{~A}$.
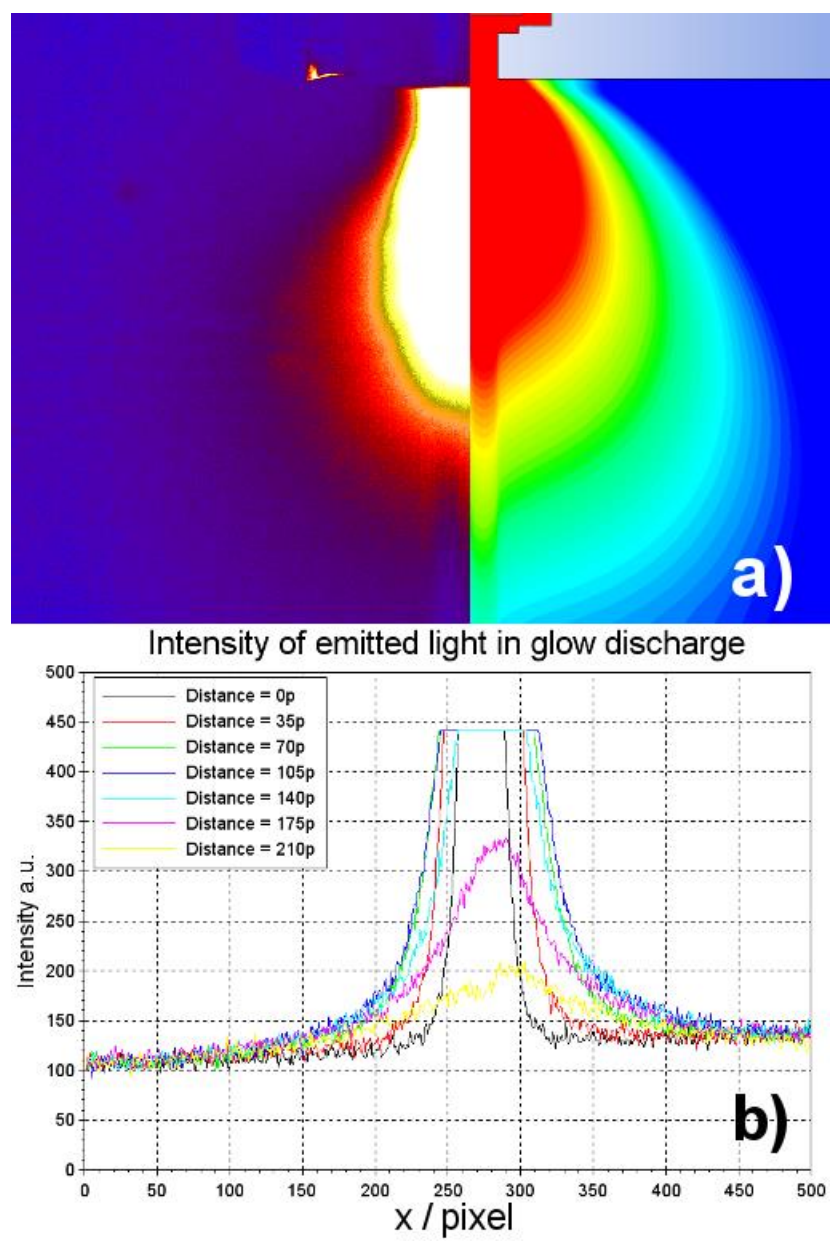

Figure 6: Color image of the gas expansion cloud $1.1 \mathrm{~ms}$ after the trigger pulse in the glow discharge (a, left side) and in the CFD simulation (a, right side). Light intensity distribution at several distances from the nozzle exit (b)

The valves gas reservoir was filled with argon at a pressure of $10 \mathrm{kPa}$. The trigger signal for the valve was used to initialize a fast camera with a line of sight perpendicular to the nozzle axis. The outflow was recorded with a framerate of $33000 \mathrm{fps}$. On the left side of Fig. $6 a$ a colored image of the light intensity emitted by the expansion cloud $1.1 \mathrm{~ms}$ after the trigger pulse is shown. The characteristic symmetrical barrel shape is clearly visible with high glow intensity in the center indicating high gas density. Shortly after the valve opening, the increased argon content in the plasma leads to discharges between valve and anode which distort the actual shape of the cloud on the images. The right side of Fig. 6a shows the resulting density distribution of the gas cloud from the CFD simulation. Experiment and simulation show a good agreement concerning the shape of the cloud. Fig. $6 \mathrm{~b}$ displays the glow intensity distribution at certain distances from the nozzle. The outline of the flow was defined where the intensity reaches half of its maximal value. This allows a measurement of the angle between the nozzle axis and the outline. The cloud expands with an angle of $54^{\circ}$ to the nozzle axis directly at the nozzle exit, decreasing along the flow until it reaches zero at the widest point of the barrel. A comparison between the CFD simulation and the experimental findings shows good agreement for both the flow shape and the outflow angle.

\section{CONCLUSION AND OUTLOOK}

An in-vessel MGI valve with a new piezoelectric drive has been built and tested. An analytical model for the $P E$ actuator was developed which fits the results of FEM simulations and experiments. A CFD model was applied to verify the assumptions made by the analytical model and to check the experimental results concerning the expansion of the gas cloud. The opening time and stroke are sufficient to perform as MGI valve. A durability test with 2000 cycles has been conducted successfully. Together with the new springdriven valves, the piezo-valve will go into operation in 2017 as part of the new in-vessel MGI system.

\section{REFERENCES}

${ }^{1}$ T.C. Hender, M.F. Johnson, C. Bachmann, N. Eidietis, S. Gerasimov, EFDAJET-R(11)01 (2011)

${ }^{2}$ G. Pautasso, C.J. Fuchs, O. Gruber, C.F. Maggi, M. Maraschek, Nucl. Fusion 47, 900 (2007)

3 D.G. Whyte, M. Bakthiari, R. Granetz, V. Izzo, J. Terry, Nucl. Materials 363,1160-1167 (2007)

${ }^{4}$ E.M. Hollmann, T.C. Jernigan, M. Groth, D.G. Whyte, D.S. Gray, Nucl. Fusion 45, 1046 (2005)

${ }^{5}$ L.R. Baylor, S.K. Combs, C.R. Foust, T.C.Jernigan, S.J. Meitner, Nucl. Fusion 49, 085013 (2009)

${ }^{6}$ S.K. Combs, S.J. Meitner, L.R. Baylor, J.B. Caughman, N. Commaux, Transactions on Plasma Science 38, 3 (2010)

7 M. Lehnen, A. Alonso, G. Arnoux, N. Baumgarten, S.A. Bozhenkov, Nucl. Fusion 51, 123010 (2011)

${ }^{8}$ A.J.Thornton, K.J. Gibson, I.T. Chapman, J.R. Harrison, A. Kirk, Nucl. Fusion 52, 063018 (2012)

${ }^{9}$ S.A. Bozhenkov, K.H. Finken, M. Lehnen, R.C. Wolf, Rev. Sci. Instrum. 78, 033503 (2007)

${ }^{10}$ C. Reux, J. Bucalossi, F. Saint-Laurent, C. Gil, P. Moreau, P. Maget, Nucl. Fusion 50, 095006 (2010)

${ }^{11}$ G. Pautasso, A. Mlynek, M. Bernert, K. Mank, A. Herrmann, Nucl. Fusion 55, $033015(2015)$

${ }^{12}$ M. Dibon, K. Mank, G. Pautasso, M. Griener, A. Herrmann, V. Mertens, R. $\mathrm{Neu}$, B. Ploeckl, V. Rohde, Spring-driven high speed valve for massive gas injection in tokamaks, to be published in Review of Scientific Instruments

$13 \mathrm{http} / / / \mathrm{www}$.noliac.com/products/actuators/plate-stacks/show/nac2023-hxx/

14 http://www.witzenmann.de/en/media/Manual_of_metal_bellows_0441e_S_9 5 115_2_04_10_20_download.pdf

${ }^{16}$ G. Pautasso, M. Bernert, M. Dibon, B. Duval, R. Dux, Plasma Phys. Control. Fusion 59, 014046 (2017) 